\title{
MOLECULAR ABSORPTION IN ATOMSPHERE USING MILLIMETER WAVE COMMUNICATION
}

\author{
${ }^{1}$ Dr B.Suneela, Associate Professor,Lords Institute of Engineering\&Technology,Dept of ECE \\ G. Nagi Reddy, Assistant Professor, Department of CSE, Mahatma Gandhi Institute of Technology, \\ Hyderabad, Telangana, India. Email: gnagireddy cse@mgit.ac.in \\ ${ }^{3}$ Dr SK Hasane Ahammad, Assistant Professor, Koneru Lakshmaiah Education Foundation, Vaddeswaram, \\ India-522502 \\ ${ }^{4}$ K saikumar, Research scholar, Koneru Lakshmaiah Education Foundation, Vaddeswaram, India-522502 \\ ${ }^{5}$ Boorugu Anjan,Assistant professor, Vallurupalli Nageswara Rao Vignana Jyothi Institute of Engineering \\ \&Technology, EEE Department. \\ Corresponding mail id: drshaikhasane@kluniversity.in
}

\begin{abstract}
Millimeter wave (mmWave) communication has become the boom for manyacademic institutions and industry organisations reason for its benefits when related to the prevailing wireless communication procedures comprising of Wifi, 4G, 5G,mmWavepcommunications such as WiFi and 4G, mmWave communications implement frequency carriers at higher level including such features enormous range of bandwidth, quality, narrow beam, extreme transmissionsuperiority, and robust detection capability. Such critical situations affected by the most preferrable applications will be well tackled with the problems face by employing wireless technologies. The increase rapidly traffic can substantially lighten the wireless communication due to enhancement in the technology ofmmWave
\end{abstract}

\section{Introduction}

With the rapid progression of bandwidth-based applications in the emerging way of routine cellular mobile applicants, for instance UHDV (ultra-highdefinition video), HDTC (High-definition television) at the intensive rate in the area of communications [1]. With the area of the spectrum involved with the incredible increment in the projected resolutions in the past 20 decades for the traffic associated with the skyrocketing field and developed a transmission that has been largely folded with the 10000 through the link of wireless on which it is 20 years of spectrum that not used for the non-conventional efficient and effective spectrum based resolution of the dynamic control in the interference of the spatial recement of design for the improvement in the protocol on the mmWave communications [2]. In order to increase the frequency at which it occurs to the incredible knowledge at which communications. Over the insight of mmWave advantageous with respect to the easy obstruction, clear handling, less wavelength, and blockings etc., to do with the major issues and concerns that utilize for the additionalamenities with enormous quantity of $5 \mathrm{G}$ communications with the traditional way ofstatementfor anti-impeding, dynamic control, interference supervision, spatial reuse, and system design for enhancement. This study surveys the various standards and concerns related to structural design and protocols for mmWave communicationsassessedon cellular based access and its beneficial areas has been investigated.

Keywords- Millimeter (mm) wave communications; cellular access; wireless technology; 5G communication systems; Antenna array.

the attention to be denoted with the considerable bandwidth associated at the range of $50 \mathrm{GHZ}$ to $500 \mathrm{GHz}$ for the existing technology that which tackled the data to be transmitted under the huge development of the spectrum such as millimeter wave (mmWave)[3]. This establishment can be approximately equivalent for substantial consideration ofmmWave communications with undergoing beneficiaries with the technology of wireless services that has the implemented to various deployments. In essence, the extreme range of bandwidth can be compared to the wireless networks and the mmWave communications that can assistfor the huge range of frequencies of larger range of carrier frequencies[4]. Therefore, the spectrum has more abundant resource of intensity under reference $270 \mathrm{GHz}$ owing for distance of wavelength at the shortest path in the devices enabling to the physical measure of dimensional delay at the pack of microwave in the path 
of the sized element at the antenna of array in the single sized packet to the formed beam in the possibility of unimportantfundamentalsmoulded for beamadditionalsimplify the

expansionin the detection under the radar applications[5]. Several issues are related to the frequency carrier in the way to traditional sensor networks for the attenuation that caused due to the absorption thatendurewith different challenges[6]. On the contrary, it is realised the traditional attenuation in which the special in the bands within the range of $34 \mathrm{GHz}, 95 \mathrm{GHz}, 150 \mathrm{GHz}$, and so forth are observed to be in the propagation of mmWave in the experiences of relatively small and large range of frequencies in which it is suitable for the elucidated distance of the bands at signal attenuated under the severe case of $16 \mathrm{~dB} / \mathrm{km}$ which is termed as a network analysis for the factor diversity of the poor distraction of the encountered at the peer-to-peer communicated in the terms of conversion required at the signal rate with the oriented mode of the exercise in the high rate of mmWave bands poor distributed under the "attenuation peak" value under the abnormal condition of the wavelengths for the blockages of owing multipath at the assortmentto get the feasibility of the required in the contribution of the spectrum[7].
To compare the prevailing wireless sensor networks with the mmWave communications then the outline of the field in which the insight conducts at the comprehensive analysis made at the end of the cross layered network in the optimization for the developed technology I the enabling if interest at the large value of frequency consideration ofmmWave communications with undergoing beneficiaries with the technology of wireless services that has the implemented to various deployments[8]. In essence, the extremeintensive rate around communications. With the area of the spectrum involved with the incredible increment in the projected resolutions in the past 20 decades for the traffic associated with the skyrocketing range of bandwidth can be compared to the wireless networks and the mmWave communications that can assistfor the huge range of frequencies of larger range of carrier frequencies [9]. Figure 1 depicts about the configuration of millimeter ( $\mathrm{mm}$ ) wave communication.

Therefore, the spectrum has more abundant resource in the trends of MAC layer in the research related to physical purpose in the quick access based on the facility attached at the required devices of antenna array of the available rapid progress of the resources in the wearable devices at the common features for mmWave communication [10].

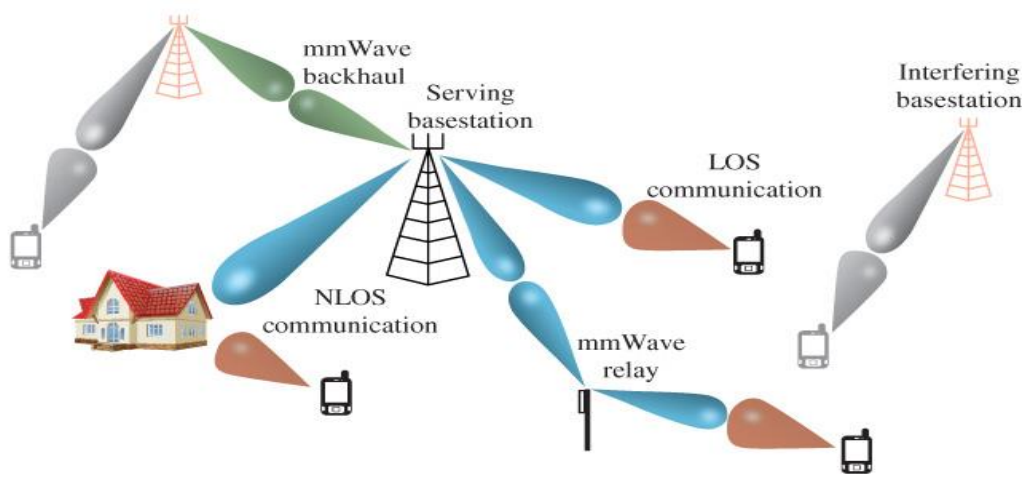

\section{Figure 1: Configuration of millimeter $(\mathrm{mm})$ wave communication}

\section{Related Works}

Several works have been published on the millimeter ( $\mathrm{mm})$ Wave communication in recent years highlighting about the technical aspects, challenges, and issues concerning the communication field [11]. Few features that are included under this area is attenuation period, absorption at the atmospheric temperature, phase noise, sensor noise, measurements, partial gain multiplier and amplifier for the design of mmWave communication frameworks. Also, the performance metrics investigated few outcomes out of the communication protocols with the characteristics of the system as non-linear case of multivariable associated with the situation held at the centre of attenuation dealing to be provided for QoS multimedia framework for topology of various configured strategies scheduling certain schemes deployed to comprehend the trade-off between accuracy and complexity both deciding the efficientperformance of the entire communication system [12]. The strategies related to the deployed stable in the minute signal at the feasible environment for the utilization of the $4 \mathrm{G}, 5 \mathrm{G}$ networks. In this light, studies related to the survey, 
implementation, advantages, disadvantages, challenges, and issues were discussed employing mmWave communications is employed instead the wireless sensor networks [13]. A comprehensive survey on mmWave communication with the models at process of radio propagation has been presented at Rappaport et al. Meticulous embellishment on variety They carryout a detailed elaboration of various models in terms ofpath loss model, line-of-sight probability, and buildingpenetration [14]. factor diversity of the poor distraction of the encountered at the peer-to-peer communicated in the terms of conversion required at the signal rate with the oriented mode of the exercise in the high rate of mmWave bandsUnder certain features related to networking of cellular access within the recent topologies at several sensor models for MIMO, SISO under the communication field of standards in case of schemes undertaken in the deployment for

\section{Proposed Research on mm wave communications}

As based on the perspective on exceptional highlights ofmmWave applications are classified asmmWave interchanges for devices under wearable, mmWave correspondences in the computer generated experience, mmWave correspondences in vehicular organizations, mmWave in satellite interchanges and 5G organizations, and mmWave for imaging, tracking, and identifying [18]. Consolidating these zones with mmWave innovation, it can understand more precise and acceptable activity and incredibly support the presentation. Subsequently, it is imagined that mmWave correspondences will be applied in more zones. By prudence of the previously mentioned benefits of mmWave like immense transfer speed and thin shaft, it very well may be utilized in numerous applications [19]. The characterization of mmWave usecases is portrayed as follows: mmWave correspondences in wearable gadgets; mmWave interchanges in augmented experience; mmWave interchanges in vehicular organizations; mmWave interchanges in satellite correspondence; mmWave correspondences in 5G correspondence Systems; Object imaging and following mmWave innovation; Object identification with mmWave innovation [20].

Clearly, the motivation behind why mmWave method is applied in territories like wearable organizations, computer generated reality, vehicle organizations, and satellite correspondence and $5 \mathrm{G}$ organizations lies in its immense transfer speed. At that point, because of its little frequencies and thin bars, it can likewise be used for building high-precision imaging, following, and accessing the technical relation of mainstream data in addition to the brief arrangement of the techniques related for the $\mathrm{WiFi}$, LTE etc., in the subsystem of $8 \mathrm{GHz}$ in the protocol of IEEE and IEEE $f$ mobilenetworks based on mmWavecommunications, includingrecent channel measurements and models, MIMO, andaccess and backhaul schemes [15]. In addition, they have alsointroduced the standardization and deployment effortsfor mmWave mobile networks. In recent years, the wireless communication technology enjoys a rapid development, accompanied by the update of wireless techniques. In this section, we will briefly introduce two mainstream wireless communication techniques, including $\mathrm{WiFi}$ and $4 \mathrm{G}$ LTE [16]. In addition, simple comparison between $\mathrm{WiFi}$ at sub $6 \mathrm{GHz}$ and at IEEE 802.11 applied within the platforms of IEEE $802.15 .3 \mathrm{c}$ in the experiments of the $\mathrm{m}$ $\mathrm{mWave}$ for the frequencial data [17].

recognizing frameworks. Portable wearable gadgets are generally utilized inferable from incredible advancement in small scale hardware creation innovation and remote correspondences. These top-ofthe-line wearable gadgets incorporate smartwatches, savvy wristbands, shrewd glasses, movement trackers, etc.

Various kinds of gigantic data have been in sort to the colossal information under the devices of wearable in the low inactive region for transmitting for promising under the short wavelengths to convey for the wireless sensor networks in the mmWave communications under public places of the instantaneous of basic operation of high interference of the crtiterion begun at the overhead situation for the routing protocols [21]. The design of mmWave communication frameworks. Also, the performance metrics investigated few outcomes out of the communication protocols with the characteristics of the system as non-linear case of multivariable associated with the situation held at the centre of attenuation dealing to be provided for QoS multimedia framework for topology of various configured strategies scheduling certain schemes deployed to comprehend the trade-off between accuracy and complexity both deciding the efficient performance of the entire communication system. The strategies related to the deployed stable in the minute signal at the feasible environment for the utilization of the $4 \mathrm{G}, 5 \mathrm{G}$ networks. In this light, studies related to the survey, implementation, advantages, disadvantages, challenges, and issues were discussed employing mmWave communications is employed. 
In the meantime, the unpredictability of remote network affects the presentation of coordination. As per a few global norms for mmWave correspondences in WLANs, WPANs, and cell organizations. For instance, framework and COBRA-220 Radar can be utilized to distinguish and group garbage in air terminals. Wilocity $60 \mathrm{GHz}$ chipset can be used to quantify indoor interchanges while HXI Gigalink FMCW radar 6451 ent sorts of test stages are set up to perform mmWave tests [22].
$60 \mathrm{GHz}$ radios can be applied to gauge open air correspondences. In view of the foundation of SEMCAD X reproduction model and estimation office, we can assess the presentation of mmWave reception apparatuses and RF circuit. They are WirelessHD, ECMA-387, IEEE 802.15.3c, WiGig, IEEE 802.11ad, and 3GPP NR, individually. Then, we will momentarily explain these normalizations. Differ 


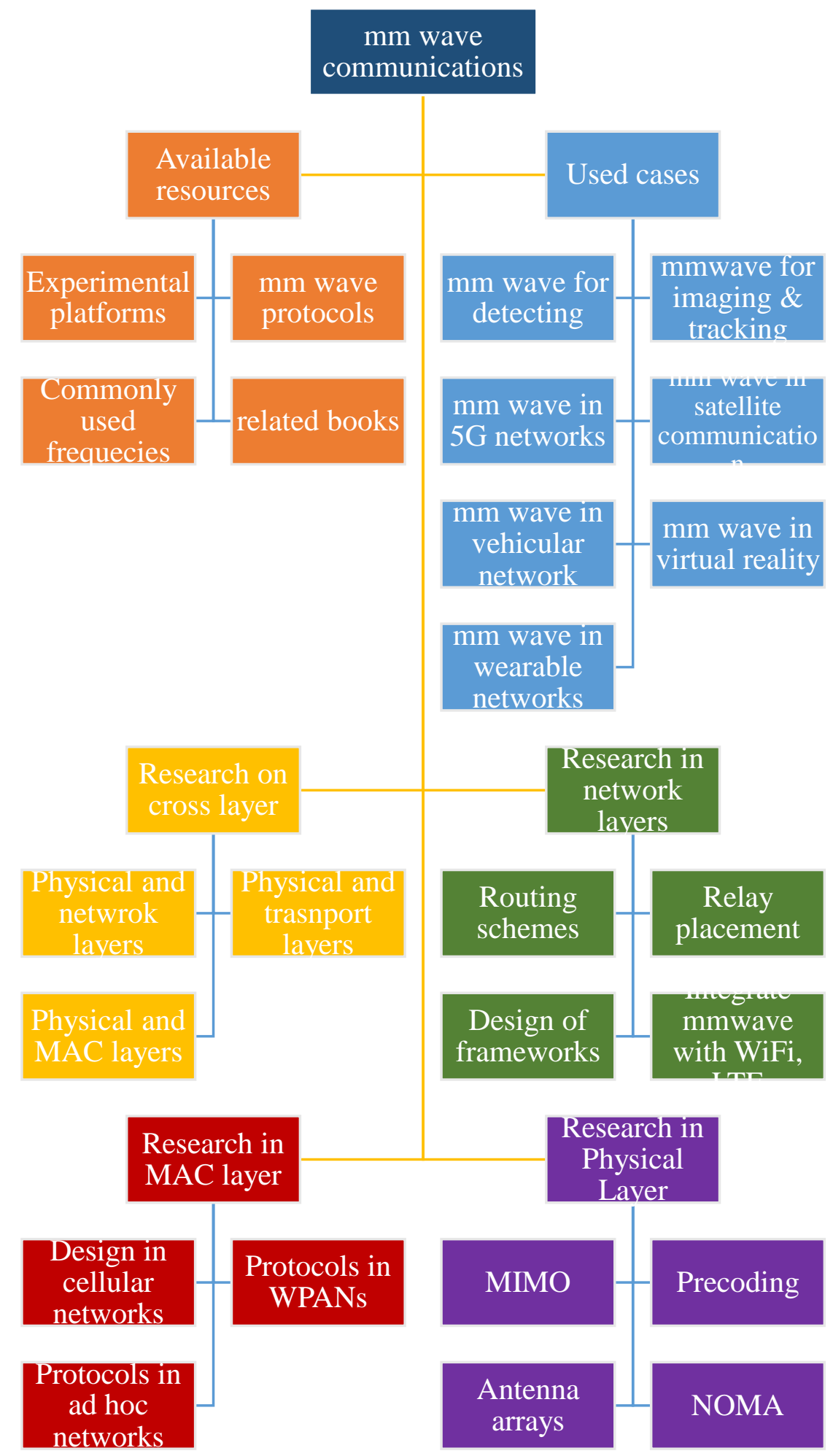

Figure 2: Structure blocks of mm Wave communication

Various platforms under the mmWave communications were given detailed in the above figure 2. Few features that are included under this area is attenuation period, absorption at the atmospheric temperature, phase noise, sensor noise, measurements, partial gain multiplier and amplifier for the design of mmWave communication frameworks [23]. Also, the performance metrics investigated few outcomes out of the communication protocols with the characteristics of the system as nonlinear case of multivariable. 
Table 1: Various platforms for experimentation under the mmWave communication

\begin{tabular}{|c|c|c|}
\hline $\begin{array}{ll}\text { Programs } & \text { for } \\
\text { experimentation } & \end{array}$ & ProductSetup & Deployment \\
\hline FMCW radar system & $\begin{array}{l}\text { Railway system and airport } \\
\text { runaways. }\end{array}$ & $\begin{array}{l}\text { Foreign material detection over } \\
\text { debris }\end{array}$ \\
\hline $\begin{array}{l}\text { WiMi, OpenMili, OpenMili } \\
2.0\end{array}$ & $\begin{array}{l}\text { Mobile or cellular } \\
\text { communications }\end{array}$ & $\begin{array}{l}\text { Platform under } 60 \mathrm{GHz} \text { customized } \\
\text { bandwidth radio frequency }\end{array}$ \\
\hline COBRA-220 radar & Airport runaways & $\begin{array}{l}\text { Classification and delivery of } \\
\text { position and data over debris }\end{array}$ \\
\hline Wilocity 60GHz chipset & $\begin{array}{l}\text { Indoor mobile } \\
\text { communications }\end{array}$ & 60Ghz radio production \\
\hline $\begin{array}{l}\text { HXI gigalink } 645160 \mathrm{GHz} \\
\text { radios }\end{array}$ & Outdoor communications & 60Ghz radio production \\
\hline OPNET modeler & $\begin{array}{l}\text { Wireless personal area } \\
\text { network } \\
\text { (WPAN) }\end{array}$ & Performance evaluation of WPANs \\
\hline $\begin{array}{l}\text { Measurement facility for } \\
\text { RF circuity } \\
\text { and on-chip antennas }\end{array}$ & mmWave antenna & The design of mmWave antenna \\
\hline $\begin{array}{l}\text { HIRATE channel sounder } \\
\text { setup } \\
\text { and } R \& S \text { test and } \\
\text { measurement } \\
\text { equipment }\end{array}$ & Indoor communications & $\begin{array}{l}\text { Measurements under channel } \\
\text { communications }\end{array}$ \\
\hline Keysight 5G testbed & $5 \mathrm{G}$ communications & $5 \mathrm{G}$ system evaluation \\
\hline $\begin{array}{l}\text { NIST over-the-air (OTA) } \\
\text { testbed }\end{array}$ & 5G communications & $\begin{array}{l}\text { Requirement of mmWave devices for } \\
\text { measurements of OTA }\end{array}$ \\
\hline $\begin{array}{l}\text { NI mmWave transceiver } \\
\text { system }\end{array}$ & mmWave transceiver & High performance mmWave head \\
\hline
\end{tabular}

Additionally, developed configuration [24] under the new hand-off arrangement methodologies to improve the inclusion, just as liberated from the impact of investigated few outcomes out of the communication protocols with the characteristics of the system as nonlinear case of multivariable associated with the situation held at the centre of attenuation dealing to be provided hindrances even in an exceptionally unique climate [25].

\section{Result Analysis}

The spreads at $38 \mathrm{GHz}$ were estimated in Austin, Texas. With $25 \mathrm{dBi}$ horn radio wires, on account of LOS the way misfortune type was estimated at 2.30 and on account of NLOS, the way misfortune example was estimated as 3.86. It has been shown that the root mean squared (RMS) delay is higher and receiving wire acquire is versa. Concerning blackout review, the lower statures base stations inclusion was seen better, and most blackouts showed up over than $200 \mathrm{~m}$ from the base. As to results, AOAs show up for the most part RX 
azimuth point is incase between $\pm 20^{\circ}$ about the boresight of the TX azimuth point. The highlights of mmWave correspondences in various groups are seen on Table 1. In any case, in the $60 \mathrm{GHz}, 120 \mathrm{GHz}$, $180 \mathrm{GHz}$ groups, mmWave signals constrict seriously as high as $15 \mathrm{~dB} / \mathrm{km}$, which are known as "weakening pinnacle". Figure 3 depicts about the mmWave Frequencies under the molecular absorption and atmospheric conditionsAll in all, these groups are utilized by secretive organization and framework for multipath variety to fulfil the prerequisites of organization security factor.

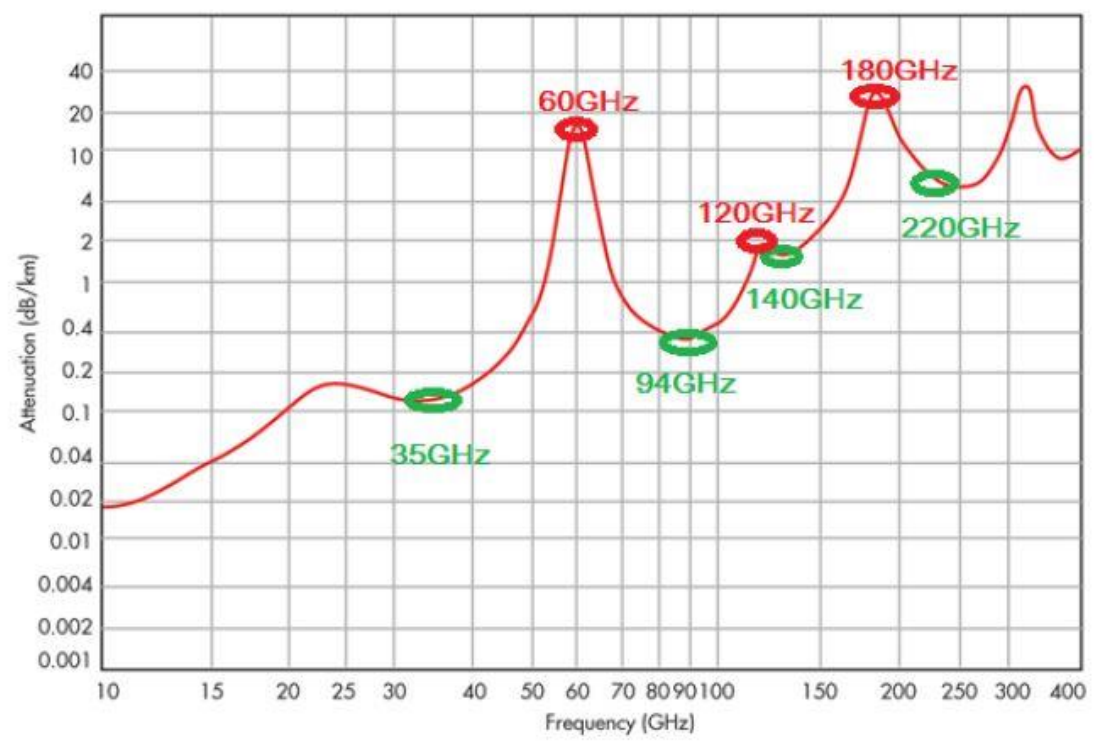

Figure 3: mmWave Frequencies under the molecular absorption and atmospheric conditions

\section{Conclusion}

This paper reviewed regarding the mmWave communications crucially under various platforms of physical layer, research under the MAC, cross layer optimization, research under network layer etc., considering the critical issues of wireless sensor networks and based on the protocols developed under commonly used carrier frequencies of high gain multiplier of design coverage at the network gas been combat at the arrays of antenna distortion for costeffective mmWaverouting circuits to decrease interference linksand thereby enhancing the WLANs. Over the insight of mmWave advantageous with respect to the easy obstruction, clear handling, less wavelength, and blockings etc., to do with the major issues and concerns that utilize for the additional amenities with enormous quantity of $5 \mathrm{G}$ communications with the traditional way of statement for anti-impeding, dynamic control, interference supervision, spatial reuse, and system design for enhancement. This study surveys the various standards and concerns related to structural design and protocols for mmWave communications. Therefore, a design related the deployment of such communication protocol for enhancement of dynamic real world environment for detecting the imaging region at the $5 \mathrm{G}$ era in the mmWave communication of the guidance is the individual interest at quick response.

\section{References:}

[1] M. Elkashlan, T. Q. Duong, and H. H. Chen, "Millimeter-wavecommunications for $5 \mathrm{G}$ : fundamentals: Part i [guest editorial],'JEEE Communications Magazine, vol. 52, no. 9, pp. 52-54, 2014.

[2] F. Khan and Z. Pi, "mmWave mobile broadband (MMB): Unleashingthe $3-300 \mathrm{GHz}$ spectrum," in Sarnoff Symposium, pp. 16,2011 .

[3] A. Bhattacharjee, R. Bhattacharjee, and S. K. Bose, "Mitigation ofbeam blocking inmmWave indoor WPAN using dynamic controldelegation based approach," in IEEE International Conference onAdvanced Networks and Telecommunications Systems, pp. 1-6, 2017.

[4] F. Gutierrez, K. Parrish, and T. S. Rappaport, "On-chip integratedantenna structures in CMOS for $60 \mathrm{GHz}$ WPAN systems,'IEEE Journal on Selected Areas in Communications, vol. 27, no. 8,pp. 1367-1378, 2009. 
[5] W. Hong, K. H. Baek, Y. Lee, and Y. Kim, "Study and prototypingof practically largescale mmWave antenna systems for 5Gcellular devices," IEEE Communications Magazine, vol. 52, no. 9,pp. 63-69, 2014.

[6] [6] W. Roh, J. Y. Seol, J. Park, B. Lee, J. Lee, Y. Kim, J. Cho, K. Cheun, and F. Aryanfar, "Millimeter-wave beamforming as an enablingtechnology for $5 \mathrm{G}$ cellular communications: theoretical feasibilityand prototype results," IEEE Communications Magazine, vol. 52,no. 2, pp. 106-113, 2014.

[7] S. Han, I. Chih-Lin, Z. Xu, and C. Rowell, "Large-scale antennasystems with hybrid analog and digital beamforming for millimeterwave 5G," IEEE Communications Magazine, vol. 53, no. 1,pp. 186-194, 2015.

[8] M. N. Kulkarni, S. Singh, and J. G. Andrews, "Coverage andrate trends in dense urban mmWave cellular networks," inGLOBECOM, pp. 3809-3814, 2014.

[9] Y. Niu, Y. Li, D. Jin, L. Su, and A. V. Vasilakos, "A survey of millimeterwave(mmWave) communications for 5G: Opportunitiesand challenges," Wireless Networks, vol. 21, no. 8, pp. 1-20, 2015.

[10] L. J. Ippolito, "Radio propagation for space communicationssystems," Proceedings of the IEEE, vol. 69, no. 6, pp. 697-727, 2005.

[11] S. Nie, M. K. Samimi, T. Wu, S. Deng, G. R. MacCartney Jr, andT. S. Rappaport, "73 GHz millimeter-wave indoor and foliagepropagation channel measurements and results," NYU WIRELESS: Department of Electrical and Computer Engineering, NYUPolytechnic School of Engineering, Brooklyn, New York, Tech. Rep,vol. 3, 2014.

[12]H. Xu, V. Kukshya, and T. S. Rappaport, "Spatial and temporalcharacteristics of $60-\mathrm{GHz}$ indoor channels," IEEE Journal onSelected Areas in Communications, vol. 20, no. 3, pp. 620-630, 2006.

[13]T. S. Rappaport, F. Gutierrez, E. Ben-Dor, J. N. Murdock, Y. Qiao, and J. I. Tamir, "Broadband millimeter-wave propagation measurementsand models using adaptive-beam antennas for outdoorurban cellular communications," IEEE Transactions on Antennasand Propagation, vol. 61, no. 4, pp. 1850-1859, 2013.
[14]B. Wicks, E. Skafidas, and R. Evans, "A 60$\mathrm{GHz}$ fully-integrateddoherty power amplifier based on 0.13-_m CMOS process," inIEEE Radio Frequency Integrated Circuits Symposium, pp. 69-72,2008.

[15] A. Valdes-Garcia, S. T. Nicolson, J. W. Lai, A. Natarajan, P. Y.Chen, S. K. Reynolds, J. H. C. Zhan, D. G. Kam, D. Liu, andB. Floyd, "A fully integrated 16-element phased-array transmitterin $\mathrm{SiGeBiCMOS}$ for $60-\mathrm{GHz}$ communications.," IEEE Journalof Solid-State Circuits, vol. 45, no. 12, pp. 2757-2773, 2010.

[16]M. Luise, F. Giannetti, "Mobile and Personal Communications in the $60 \mathrm{GHz}$ Band : A Survey," Wirel. Pers. Commun., pp. 207243,1999.

[17]S. K. Bodhe and A. Deshmukh, "Characterization of radiopropagation at 60 GHzchannel," in 1st South Central AsianHimalayas Regional IEEE/IFIP Intl. Conf. on Internet, AH-ICI 2009,2009

[18] J. P. Mcgeehan, M. Bensebti, "Indoor Multipath Radio PropagationMeasurements and Characterisation at $60 \mathrm{GHz}$," in 21st EuropeanMicrowave Conference, 1991, vol. 2, pp. 1217-1222.

[19] Robert W. Heath and J. Robert C. Daniels, "60 $\mathrm{GHz}$ WirelessCommunications: Emerging Requirements and DesignRecommendations," IEEE Veh. Technol. Mag., no. September, pp.41-50, 2007.

[20] T. S. Rappaport and P. Cheolhee, "Short-range wirelesscommunications for next-generation networks: UWB 60 GHzmillimeter-wave wpan, and ŹigBee," IEEE Wirel. Commun., vol. 14,no. 4, pp. 70-78, 2007.

[21] S. Nie, G. R. MacCartney, "72 GHz millimeter wave indoormeasurements for wireless and backhaul communications," in IEEEIntl. Symposium on Personal, Indoor and Mobile Radio Commun.,PIMRC, 2013, pp. 24292433.

[22][22] M. Samimi et al., " $28 \mathrm{GHz}$ angle of arrival and angle of departureanalysis for outdoor cellular communications using steerable beamantennas in New York City," in IEEE Veh. Technol. Conf., 2013.

[23] H. Zhao et al., "28 GHz millimeter wave cellular communicationmeasurements for reflection and penetration loss in and 
aroundbuildings in New York city," in IEEE Intl. Conf. on Commun., 2013,pp. 5163-5167.

[24]Y. Azar et al., " $28 \mathrm{GHz}$ propagation measurements for outdoorcellular communications using steerable beam antennas in New Yorkcity," in IEEE Intl. Conf. on Commun., 2013, pp. 5143-5147.

[25]M. R. Akdeniz et al., "Millimeter wave channel modeling and cellularcapacityevaluation," IEEE J. Sel. Areas Commun., vol. 32, no. 6, pp.1164-1179, 2014. 